\title{
Study of changes in catalase activity in rat brain under mechanical influence on hemato- ophthalmic barrier
}

\author{
Anatoly Devyatkin ${ }^{1}$, Olga Pavlova ${ }^{2,3,}{ }^{*}$, Olga Gulenko ${ }^{2}$, and Pavel Boriskin ${ }^{1}$ \\ ${ }^{1}$ Kazan State Academy of Veterinary Medicine named after N.E. Bauman, 420074, st. Siberian tract, \\ 35, Kazan, Republic of Tatarstan, Russia \\ ${ }^{2}$ Samara State University of Railways, 443077, Svobody st., 2B, Samara, Russia \\ ${ }^{3}$ Private institution educational organization of higher education "Medical University \\ "Reaviz",443099, st.Chapaevskaya, 227, Samara, Russia
}

\begin{abstract}
Hemato-ophthalmic barrier protects nervous system tissues from adverse factors. Against the background of inflammatory process caused by mechanical trauma of the eye, large molecules are able to penetrate the eyeball. At present, the mechanism of restoring the homeostatic balance of the body under oxidative stress caused by mechanical trauma of the eye is poorly studied. The aim of the study was to study the dynamics of catalase activity in rat brain tissue under oxidative stress caused by mechanical influence on hemato-ophthalmic barrier. Materials and methods: the study was conducted on white male rats of six months of age in the amount of 150 pieces. The activity of catalase in brain tissue was studied before the experiment, as well as on the 1st, 3rd, 5th, 7th and 14th day of the experiment using the standard Korolyuk M.A. method. The obtained digital material was statistically processed by non-parametric statistical analysis. Conclusion: The activity of catalase in the rat brain under oxidative stress caused by mechanical action on the hematoophthalmic barrier is most effectively stabilized in the standard therapy of mechanical eye injury with the addition of quercetin in the form of injections.
\end{abstract}

\section{Introduction}

Oxidative stress is a state of the organism that develops against the background of overproduction of free radicals, when the effectiveness of antioxidant protection is violated [1-2]. Such a state leads to the development of a wide range of pathologies [3]. Due to the high content of lipids and fatty acids capable of oxidizing with the formation of toxic compounds and intensive oxygen consumption, the largest target for free radicals is nervous system tissues [4]. The greater the functional load on the nervous system, the more oxygen is needed, and therefore, more free radicals are formed. However, nervous system tissues, in particular the brain, lack the enzymes that help deactivate the active oxygen forms.

\footnotetext{
*Corresponding author: casiopeya13@mail.ru
} 
Hemato-encephalic and hemato-ophthalmic barriers protect nervous system tissues from negative effects, but at the same time prevent the action of natural antioxidants. Under prolonged oxidative stress, it is possible that cells accumulate microgly of inflammatory mediators, which in turn contribute to the production of active oxygen forms, providing a cyclical pathological processes [5-10]. At the same time, the functioning of protective barriers is impaired, accompanied by the penetration of neurotoxins that contribute to the development of neurodegenerative diseases [11-18]. Discovery of molecular mechanisms of such pathogenetic processes is the basis for the development of effective therapeutic approaches.

Thus, the aim of our study was to study the dynamics of catalase activity in rat brain tissue under oxidative stress caused by mechanical action on the hemato-ophthalmic barrier.

In order to realize the set goal it was necessary to solve the following tasks: to determine the changes of catalase activity in brain tissue of intact rats and rats with induced oxidative stress caused by mechanical influence on hemato-ophthalmic barrier; to reveal the effectiveness of oxidative stress relief when applying different methods of eye injury treatment.

\section{Materials and methods}

The study was carried out on white pedigree matured healthy male rats of six months of age, weighing 200-220 g in the amount of 150 pieces, which were contained in the vivarium under standard conditions.

All animals were divided into 5 groups of thirty rats in each group. Animals of group 1 are intact rats. Animals of the 2, 3, 4 and 5 groups were applied in a special fixation device - a penetrating wound to the right and left eye in the limb area with the help of a microblade under local anesthesia with the use of two percent solution of novocaine. The cornea, sclera and vascular tract were injured. Animals in 2 groups were kept without treatment. Animals of the 3 groups were treated with standard therapy for mechanical eye injury, which included daily injection of subcutaneous dexamethasone phosphate of $0.1 \mathrm{mg} / \mathrm{kg}$ of body weight once a day, sodium diclofenac of $0.5 \mathrm{mg} / \mathrm{kg}$ once a day, gentamicin sulfate of 1.5 $\mathrm{mg} / \mathrm{kg} 2$ times a day. Also dexamethasone 4 times a day and mesaton 2 times a day (in titration according to animal weight) were installed in the damaged eye. This treatment scheme was carried out within a week after the injury. Then during the month continued the installation of dexamethasone solution on a descending scheme $(3,2,1$ time a day for 2 days each mode) and Nevanaka 4 times a day (or broxinaka 1 time a day). Animals of the 4 groups also received standard therapy for eye injury, but were added intraperitoneal injections of flavonoid quercetin (ICN, USA) in a dose of $5 \mathrm{mg} / \mathrm{kg}$ mass. Animals of group 5 received only intraperitoneal injections of quercetin in a dose of $5 \mathrm{mg} / \mathrm{kg}$ mass. The therapy of mechanical eye injury lasted 14 days. During the experiment, all animals were on a standard vivarium diet with free access to water and food. All groups of rats were involved in the experiment simultaneously, which excludes the influence of external temperature, climatic and other factors on the activity of the studied enzyme in control and experimental groups [19].

The activity of catalase in brain tissue was studied before the experiment and on the 1st, 3rd, 5th, 7th, and 14th day of the experiment using the standard Korolyuk M.A. method [19]. In accordance with ethical norms, rats were killed by decapitation method under ether anesthesia and then brain was extracted, which was washed with physiological solution and frozen. Then, by mechanical grinding of brain tissue weighing $1 \mathrm{~g}$ with $9 \mathrm{ml}$ tris-buffer $(\mathrm{pH}$ 7.4) homogenates were prepared in automatic homogenizer with the speed of $5000 \mathrm{rpm}$ in a double-walled vessel permanently cooled by running water [20]. 
The study was carried out in accordance with the rules of laboratory practice in the Russian Federation: Order of the Ministry of Health of the USSR No. 755 dated 12.08.1977; Order of the Ministry of Health of the Russian Federation No. 267 dated 19.06.2003; Law "On protection of animals from cruelty", Chapter V, Art. 104679-GD dated 01.12.1999. The conclusion of the Committee on Bioethics at the "Medical University "Reaviz" No. 163 dated 29 August 2019 was received for the experiment.

The obtained digital material was subjected to statistical processing by means of nonparametric statistical analysis in order to establish authenticity of differences in the studied groups using Valda-Volfovitz, Kolmogorov-Smirnov and Manna-Whitney criteria.

\section{Results of the study}

The dynamics of catalase (Me) activity in rat brain tissue is shown in Figure 1.

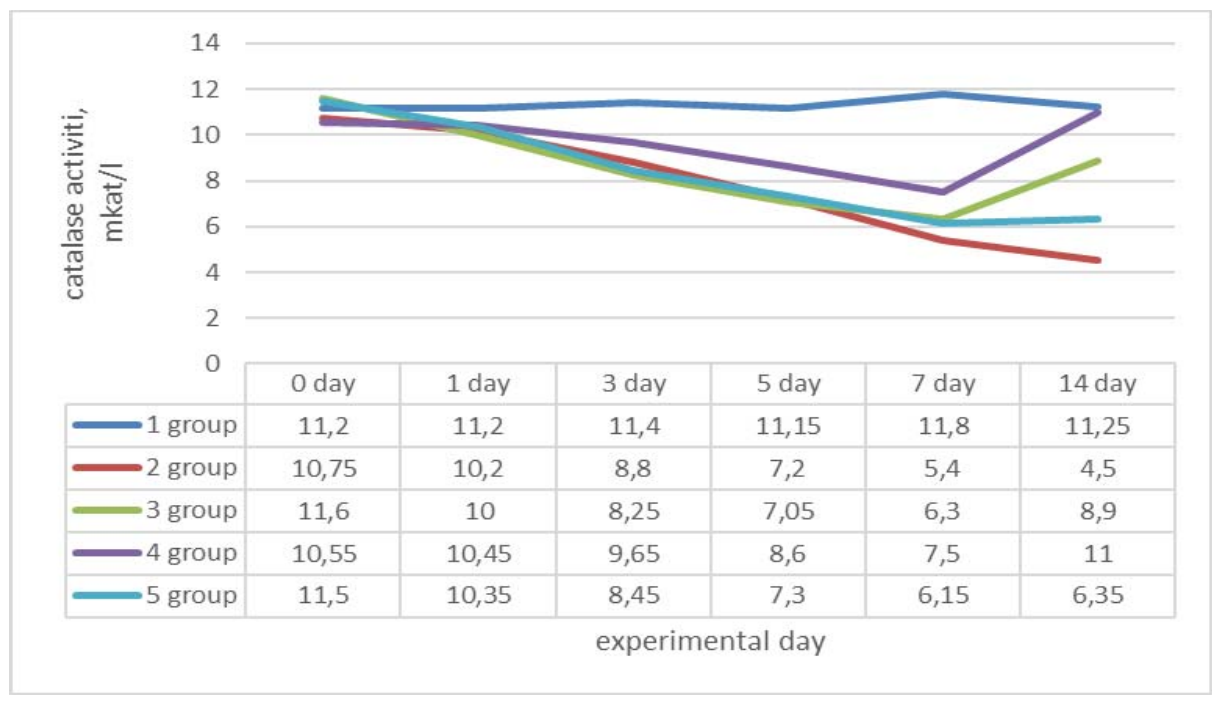

Fig. 1. Dynamics of catalase activity in rat brain tissue under oxidative stress caused by mechanical influence on hemato-ophthalmic barrier.

According to the presented picture, intact rats (group 1) catalase activity was approximately at the same level within the physiological norm throughout the experiment. In animals of the 2nd group with experimentally induced oxidative stress, by traumatizing the hemato-ophthalmic barrier, the activity of catalase decreased significantly throughout the whole experiment.

The animals of the third group also showed a decrease in catalase activity up to 7 days of experience, but by 14 days the enzyme activity increased significantly and was slightly higher than the third day of experience, i.e., it aspired to the physiological norm.

In the 4th group of rats, a less intensive decrease in catalase activity was observed up to 7 days of experience, and by the 14th day the enzyme activity almost reached the physiological norm.

In the fifth group of animals a decrease in catalase activity was observed intensively up to 7 days of experience, and by 14 days the enzyme activity tended to increase.

The obtained results were processed by non-parametric statistical analysis to determine the reliability of differences in the studied groups using Valda-Volfovitz, KolmogorovSmirnov and Manna-Whitney criteria. 
It was found that catalase activity in brain tissue in animals of the 2nd group from the first day of experience differed significantly from that in intact rats:

- 1 day - criterion of Mann-Whitney $U=192,500, Z=3,807$ at $p=0,000141$; criterion of Kolmogorov-Smirnov Max Neg Differnc $=0,0000$, Max Pos Differnc $=0,4667$, at $\mathrm{p}<$ 0,005 ; criterion of Valda-Wolfowitz $Z=-0,7812$ at $\mathrm{p}$-level $=0,434659, \mathrm{Z}$ adjstd $=0,6510$ at p-level $=0,515022$;

- 3, 5, 7 and 14 days - Mann-Whitney criterion $U=0,000, Z=6,653$ at $p=0,000000$; Kolmogorov-Smirnov Max Neg Differnc $=0,0000$, Max Pos Differnc $=1,0000$, at $\mathrm{p}<$ 0,001 ; Valda-Wolfowitz criterion $Z=-7,5520$ at $p$-level $=0,000000, Z$ adjstd $=7,4218$ at $p$ level $=0,000000$.

In the second group, starting from the first day of experience, the activity of catalase significantly differs to a lesser extent from that of intact rats, as violation of the hematoophthalmic barrier leads to the intensification of oxidative stress.

The activity of catalase in the brains of animals of group 3 also from the first day of experience differed significantly from that of intact rats:

- 1 day - Mann-Whitney criterion $U=121,500, Z=4,857$ at $p=0,000001$; KolmogorovSmirnov Max Neg Differnc $=0,0000$, Max Pos Differnc $=0,5667$, at $p<0,001$; ValdaWolfowitz criterion $Z=-3,3854$ at $p$-level $=0,000711, Z$ adjstd $=3,2552$ at $p$-level $=$ 0,001133

- 3, 5 and 7 days - Mann-Whitney criterion $U=0,000, Z=6,653$ at $p=0,000000$; Kolmogorov-Smirnov Max Neg Differnc $=0,0000$, Max Pos Differnc $=1,0000$, at $p<$ 0,001; Valda-Wolfowitz criterion $Z=-7,5520$ at $p$-level $=0,000000, Z$ adjstd $=7,4218$ at $p$ level $=0,00000$;

- 14-day Mann-Whitney Criterion $U=4,500, Z=6,586$ at $p=0,000000$; Max Neg Differnc, Max Pos Differnc, Kolmogorov-Smirnov Criterion $=0,0000$, Max Pos Differnc $=0,9667$ at $\mathrm{p}<0,001$; Valda-Wolfowitz Criterion $\mathrm{Z}=-7,0312$ at $\mathrm{p}$-level $=0,000000, \mathrm{Z}$ adjstd $=6,9010$ at $\mathrm{p}$-level $=0,00000$.

In animals of the third group with induced oxidative stress and its reduction by applying the standard therapy of mechanical trauma of the eye catalase activity in the brain tissue is also significantly different from that of intact rats throughout the whole experience, but in general, by 14 days the activity of the enzyme tends to the physiological norm, which indicates inactivation of oxidative stress.

The activity of catalase in the brain of animals of group 4 in zero, as well as 1, 3, 5 and 7 days of experience differed significantly from that of intact rats:

- 0 day - criterion of Mann-Witney $U=292,500, Z=2,329$ at $p=0,019884$; criterion of Kolmogorov-Smirnov Max Neg Differnc $=-0,0667$, Max Pos Differnc $=0,3667$, at $\mathrm{p}<$ 0,050 ; Valda-Wolfowitz criterion $Z=-0,5208$ at $p$-level $=0,602485, Z$ adjstd $=0,3906$ at $p$ level =0,696076;

- 1 day - Mann-Whitney criterion $U=257,500, Z=2,846$ at $p=0,004427$; KolmogorovSmirnov Max Neg Differnc = -0,0333, Max Pos Differnc $=0,3333$, at $p<0,10$; WaldWolf criterion $Z=0,0000$ at $p$-level $=1,000000, Z$ adjstd $=-0,1302$ at $p$-level $=0,896402$;

- 3-day Mann-Whitney criteria $U=11,000, Z=6,490$ at $p=0,000000$; Kolmogorov-Smirnov Max Neg Differnc $=0,0000$, Max Pos Differnc $=0,8667$, at $p<0,001$; Valda-Wolfowitz criteria $Z=-5,4687$ at $p$-level $=0,000000, Z$ adjstd $=5,3385$ at $p$-level $=0,000000$;

- 5, and 7 days - Mann-Whitney criterion $U=0,000, Z=6,653$ at $p=0,000000$; KolmogorovSmirnov Max Neg Differnc $=0,0000$, Max Pos Differnc $=1,0000$, at $p<0,001$; ValdaWolfowitz criterion $Z=-7,5520$ at $\mathrm{p}$-level $=0,000000, \mathrm{Z}$ adjstd $=7,4218$ at $\mathrm{p}$-level $=$ 0,000000 ;

In the 4th group of rats, the activity of catalase on the 14th day of the experiment does not differ from intact animals, which indicates a more effective treatment of oxidative stress 
by applying the standard therapy of mechanical injury to the eye with the addition of quercetin.

The activity of catalase in brain tissue in animals of group 5 from the first day of the experiment differed significantly from that of intact rats:

- 1 day - Mann-Whitney criterion $U=267,500, Z=2,706$ at $p=0,006820$; KolmogorovSmirnov Max Neg Differnc $=0,0000$, Max Pos Differnc $=0,3333$, at $p<0,10$; ValdaWolfowitz criterion $\mathrm{Z}=0,2604$ at $\mathrm{p}$-level $=0,794544, \mathrm{Z}$ adjstd $=0,1302$ at $\mathrm{p}$-level $=$ 0,896402 ;

- 3, 5, 7 and 14 days - Mann-Whitney criterion $U=0,000, Z=6,653$ at $p=0,000000$; Kolmogorov-Smirnov Max Neg Differnc $=0,0000$, Max Pos Differnc $=1,0000$, at $\mathrm{p}<$ 0,001 ; Valda-Wolfowitz criterion $Z=-7,5520$ at $\mathrm{p}$-level $=0,00000, \mathrm{Z}$ adjstd $=7,4218$ at $\mathrm{p}$ level $=0,000000$.

In the fifth group of animals also observed a decrease in the activity of catalase on the background of oxidative stress, and the index throughout the experience differs from that of rats, which indicates that the therapy of mechanical trauma to the eye only with quercetin is not effective.

\section{Conclusions}

Mechanical trauma of the eye is a stimulant of oxidative processes in the body, as toxic products of autolysis of damaged tissues enter the blood and intensify oxidation and production of free radicals. Catalase, as one of the enzymes of the antioxidant system, is actively involved in the fight against the resulting particles and its activity in tissues is reduced. Quercetin is also effective in suppressing singlet oxygen and activating antioxidant enzymes in the body [21-22].

In our study on rats it was found that in addition to the standard therapy of mechanical eye injury injection of quercetin, quercetin's catalase activity in the brain after a fall induced oxidative stress increases to almost the physiological norm values by 14 days, which indicates the effective elimination of oxidative stress.

The activity of catalase in the rat brain under oxidative stress caused by mechanical action on the hemato-ophthalmic barrier is most effectively stabilized in standard therapy of mechanical eye injury with the addition of quercetin in the form of injections.

\section{References}

1. M. A. Lutsky, et al., Successes of modern natural science, 12-1, 20-23 (2014)

2. O. N. Pavlova, S. A. Simakova, Medico-physiological problems of human ecology: materials of the IV All-Russian Conference with international participation, 244-246 (2011)

3. E. E. Vasenina, Modern therapy in psychiatry and neurology, 3-4, 39-46 (2013)

4. V. A. Kotsur, et al., Proceedings of XIII International Scientific-Practical Conference. Magnitogorsk: G.I. Nosov State Technical University Publishing House, 163-164 (2018)

5. V. S. Rogovsky, et al., Zhurn. experimental and clinical pharmacology, 75(9), 37-41 (2012)

6. P. Walton, et al., Biochim Biophys Acta Mol Cell Res, 1864, 1833-1843 (2017)

7. A. Karich, et al., Journal of Molecular Catalysis B: Enzymatic, 134, 238-246 (2016)

8. H. Medina-Gomez, et al., Microbial Pathogenesis, 125, 93-95 (2018) 
9. N. Mamadalieva, et al., Bulletin of Novosibirsk state pedagogical University, 1, 222231 (2014)

10. S. V. Uglanova, Vestnik of Moscow University, 51(3), 227-234 (2010)

11. P. M. Carvey, et al., Eur. J. Neurosci., 22, 1158-1168 (2005)

12. E. E. Vasenina, O. S. Levin, Neuroprotective therapy., 3-4, 39-46 (2013)

13. C. Akateh, et al., Journal of Surgical Research, 238, 152-163 (2019)

14. C. Glorieux, et al., Free Radical Biology and Medicine, 87, 84-97 (2015)

15. H. Sepasi Tehrani, et al., Progress in Biophysics and Molecular Biology, 140, 5-12 (2018)

16. C. Heit, et al., Free Radical Biology and Medicine, 103, 48-56 (2017)

17. A. Grigoras, Biochemical Engineering Journal, 117, 1-20 (2017)

18. D. Maksimovich, et al., Internauka, (12-1), 10-12 (2017)

19. H. Sies, et al., Annu. Rev. Biochem., 86, 715-748 (2017)

20. F. Kyota, et al., Medicine and cellular longevity, 1, 9 (2012)

21. I. N. Mikheitseva, et al., Ophthalmological Journal, 2, 62-67 (2015)

22. A. S. Balakina, et al., Voprosy etiquette, 2, 14-22 (2017) 